\title{
Efficiency in uncertain cooperative games
}

Citation for published version (APA):

Peters, H. J. M., Bossert, W., \& Derks, J. (2001). Efficiency in uncertain cooperative games. METEOR, Maastricht University School of Business and Economics. METEOR Research Memorandum No. 008 https://doi.org/10.26481/umamet.2001008

Document status and date:

Published: 01/01/2001

DOI:

10.26481/umamet.2001008

Document Version:

Publisher's PDF, also known as Version of record

\section{Please check the document version of this publication:}

- A submitted manuscript is the version of the article upon submission and before peer-review. There can be important differences between the submitted version and the official published version of record.

People interested in the research are advised to contact the author for the final version of the publication, or visit the DOI to the publisher's website.

- The final author version and the galley proof are versions of the publication after peer review.

- The final published version features the final layout of the paper including the volume, issue and page numbers.

Link to publication

\footnotetext{
General rights rights.

- You may freely distribute the URL identifying the publication in the public portal. please follow below link for the End User Agreement:

www.umlib.nl/taverne-license

Take down policy

If you believe that this document breaches copyright please contact us at:

repository@maastrichtuniversity.nl

providing details and we will investigate your claim.
}

Copyright and moral rights for the publications made accessible in the public portal are retained by the authors and/or other copyright owners and it is a condition of accessing publications that users recognise and abide by the legal requirements associated with these

- Users may download and print one copy of any publication from the public portal for the purpose of private study or research.

- You may not further distribute the material or use it for any profit-making activity or commercial gain

If the publication is distributed under the terms of Article $25 \mathrm{fa}$ of the Dutch Copyright Act, indicated by the "Taverne" license above, 


\title{
Efficiency in Uncertain Cooperative Games*
}

\author{
WALTER BOSSERT \\ Département de Sciences Economiques and C.R.D.E. \\ Université de Montréal \\ C.P. 6128, succursale Centre-ville \\ Montréal QC H3C 3J7 \\ Canada \\ e-mail: Walter.Bossert@umontreal.ca \\ JEAN DERKS \\ Department of Mathematics \\ University of Maastricht \\ P.O. Box 616 \\ 6200 MD Maastricht \\ The Netherlands \\ e-mail: jean.derks@math.unimaas.nl \\ HANS PETERS \\ Department of Quantitative Economics \\ University of Maastricht \\ P.O. Box 616 \\ 6200 MD Maastricht \\ The Netherlands \\ e-mail: h.peters@ke .unimaas.nl
}

May 2001

* Financial support from the Centre de Recherche et Développement en Economique of the Université de Montréal and the Social Sciences and Humanities Research Council of Canada is gratefully acknowledged. 


\begin{abstract}
A contingent contract in a transferable utility game under uncertainty specifies an outcome for each possible state. It is assumed that coalitions evaluate these contracts by considering the minimal possible excesses. A main question of the paper concerns the existence and characterization of efficient contracts. It is shown that they exist if and only if the set of possible coalitions contains a balanced subset. Moreover, a characterization of values that result in efficient contracts in the case of minimally balanced collections is provided. Journal of Economic Literature Classification Number: C71.
\end{abstract}

Keywords: Transferable Utility Games, Uncertainty, Balanced Collections. 


\section{Introduction}

Consider a group of players facing a cooperative game-theoretic situation of which the data are uncertain. To focus on an example, suppose the players are firms that decide to cooperate, but the demands for the various goods that they produce fluctuate over time, and so do the profits that each coalition of firms could obtain. In order to keep cooperation guaranteed the firms could try to design a contract stating the profit shares of the firms for all contingencies that might arise. For the evaluation of such a contract a firm can use some criterion for decision making under uncertainty. In the present paper it will be assumed that firms wish to be on the safe side and evaluate a contract by its worst case. The central questions that we raise are the following. Can we characterize the contracts that are efficient with respect to this criterion? Do such contracts always exist? Is it possible to assign a solution (for example, a profit sharing agreement) to each certain situation such that this results in contingent contracts under uncertainty that are efficient?

We will study these questions in the context of transferable utility (TU) games, where each coalition is characterized by the payoff it can obtain by cooperation. In line with the above example one may think of these payoffs as representing monetary amounts, such as profits. A cooperative game under uncertainty is a pair of TU games: one of these will be the true game. For an associated pair of payoff vectors, each coalition calculates its minimum gain and uses the resulting numbers to rank such pairs. When we say 'each coalition' we mean each coalition in a predestined set of possible coalitions, since in most settings it is natural to assume that not every coalition can form. We look for efficient (Pareto undominated) pairs of vectors. The associated domination relation can be formulated also as a property of payoff vectors in a certain game. Precise definitions of the concepts used here are presented in Section 2.

Section 3 is devoted to studying this domination relation for payoff vectors in certain TU games. The connection with the core and the anti-core is established, and undominated payoff vectors are characterized in terms of balanced sets of coalitions. The results in this section are originally motivated by the analysis of efficient contracts under uncertainty, but they are of independent interest as well.

In Section 4 we consider values - solutions that assign payoff vectors to TU games that always yield an efficient pair in a cooperative game under uncertainty. A complete characterization of these solutions is obtained for the case where the set of allowed coalitions is a minimally balanced collection. This characterization involves monotone paths and, 
thus, a link is established to similar results in the pure bargaining context (Bossert, Nosal and Sadanand, 1996; Bossert and Peters, 2001a,b). Furthermore, an existence result for weakly balanced (but not necessarily minimally balanced) collections is proven. As a byproduct, we also obtain existence of undominated allocations in certain games. Moreover, the results on values can easily be extended to more than two possible states.

We conclude, in Section 5, by briefly discussing a stronger version of the undominatedness property and a related open question.

\section{Preliminaries}

Throughout, $N=\{1, \ldots, n\}$ is the set of players. Our convention for vector inequalities is the following. For $x, y \in \mathbb{R}^{N}, x \geq y$ if $x_{i} \geq y_{i}$ for all $i \in N$, and $x>y$ if $x_{i}>y_{i}$ for all $i \in N$.

A transferable utility game (TU game) is a pair $(\mathcal{B}, v)$, where $\mathcal{B}$ is a nonempty set of nonempty strict subsets of $N$, and $v: \mathcal{B} \cup\{N\} \rightarrow \mathbb{R}$ is a mapping assigning to each coalition $S \in \mathcal{B} \cup\{N\}$ its worth $v(S)$. Let $\mathcal{G}^{\mathcal{B}}$ denote the set of all such TU games. If no confusion is likely we write $v$ instead of $(\mathcal{B}, v)$ and $\mathcal{G}$ instead of $\mathcal{G}^{\mathcal{B}}$. It is understood that whenever we talk about coalitions we mean elements of $\mathcal{B} \cup\{N\}$, unless stated otherwise.

For every $v \in \mathcal{G}$ denote by $F(v)$ the set $F(v):=\left\{x \in \mathbb{R}^{N}: x(N)=v(N)\right\}$, where we adopt the notation $x(S)=\sum_{i \in S} x_{i}$ for every coalition $S$. Vectors in $\mathbb{R}^{N}$ are called allocations, and vectors in $F(v)$ are called efficient allocations. ${ }^{1}$

A set $B \subseteq \mathcal{B}$ is balanced if it is either empty or there are positive numbers $\lambda_{S}(S \in B)$ with $\sum_{S \in B: i \in S} \lambda_{S}=1$ for every $i \in N$. The set $B$ is minimally balanced if it is balanced and contains no balanced subset other than the empty set and $B$ itself. A set of coalitions $B \subseteq \mathcal{B}$ is said to be weakly balanced if it contains a nonempty balanced subset.

For a subset $S \subseteq N$, the indicator vector $e^{S}$ is defined by $e_{i}^{S}=1$ if $i \in S$ and $e_{i}^{S}=0$ if $i \notin S$.

Our notion of dominance is defined as follows.

Definition 1 For $w, w^{\prime} \in \mathcal{G}$ and $q, z \in F(w), q^{\prime}, z^{\prime} \in F\left(w^{\prime}\right)$ we say that the pair $\left(q, q^{\prime}\right)$ dominates the pair $\left(z, z^{\prime}\right)$ if for every $S \in \mathcal{B}$ we have

$$
\min \left\{q(S)-w(S), q^{\prime}(S)-w^{\prime}(S)\right\}>\min \left\{z(S)-w(S), z^{\prime}(S)-w^{\prime}(S)\right\} .
$$

If there is no pair $\left(q, q^{\prime}\right)$ dominating $\left(z, z^{\prime}\right)$ then the latter pair is called undominated in $\left(w, w^{\prime}\right)$.

\footnotetext{
${ }^{1}$ In the literature, efficient allocations are also called 'preimputations.'
} 
The above definition of dominance can be generalized by considering an arbitrary number of possible states when applying the maximin criterion to games under uncertainty. We return to this issue in Section 4, where we demonstrate that, for the purposes of finding domination efficient values, the above formulation in terms of two possible states does not involve any loss of generality.

The purpose of this paper is to identify undominated pairs for pairs of games $\left(w, w^{\prime}\right) \in$ $\mathcal{G} \times \mathcal{G}$. The interpretation is as follows. Suppose that it is uncertain whether the true game will be $w$ or $w^{\prime}$. A pair $\left(z, z^{\prime}\right)$ with $z \in F(w), z^{\prime} \in F\left(w^{\prime}\right)$, can be seen as a contingent contract among the players written before the uncertainty is resolved. Let $\mathcal{B}$ be the relevant set of coalitions, that is, the coalitions that would have the power to upset the contract. We exclude $N$ since by efficiency the payoff to $N$ is fixed. If these coalitions wish to be on the safe side or, alternatively, are pessimistic in the sense of being worst-case maximizers, then undominatedness of the pair $\left(z, z^{\prime}\right)$ means that there is no contract that is better for all coalitions, evaluated in terms of the gains over the coalitional worths. ${ }^{2}$ Alternatively, one could demand that there is no contract that is at least as good for all coalitions and strictly better for at least one coalition. This possibility is discussed in Section 5.

Before proceeding with a closer examination of the domination relation in Definition 1 , we adopt the notational convention to consider a vector $x \in \mathbb{R}^{N}$ simultaneously as an additive game, where coalition $S \in \mathcal{B} \cup\{N\}$ has worth $x(S)$. Further, comparisons of games should be considered coalition-wise: for a subset $B$ of coalitions, $v \leq w$ on $B$ means $v(S) \leq w(S)$ for every $S \in B$, and $v<w$ on $B$ means $v(S)<w(S)$ for all $S \in B$. The minimum of two games, $\min \{v, w\}$, on $B$ is the game in which each coalition $S \in B$ has worth $\min \{v(S), w(S)\}$. Finally, a sidepayment is a vector $y \in \mathbb{R}^{N}$ with $y(N)=0$.

Note that the pair $\left(z, z^{\prime}\right)$ is undominated in $\left(w, w^{\prime}\right) \in \mathcal{G} \times \mathcal{G}$ if, and only if, for all $q \in F(w), q^{\prime} \in F\left(w^{\prime}\right)$,

$\min \left\{q-w, q^{\prime}-w^{\prime}\right\} \geq \min \left\{z-w, z^{\prime}-w^{\prime}\right\} \Rightarrow \min \left\{q-w, q^{\prime}-w^{\prime}\right\} \ngtr \min \left\{z-w, z^{\prime}-w^{\prime}\right\}$

on $\mathcal{B}$. In other words, if there is a weak inequality on $\mathcal{B}$, there should be at least one coalition $S$ for which equality holds. Letting $v=w-w^{\prime}, x=z-z^{\prime}$, and writing $y=q-z$ and $y^{\prime}=q^{\prime}-z^{\prime}$, this is equivalent to the following statement. For all sidepayments $y, y^{\prime}$,

$$
\min \left\{x+y, v+y^{\prime}\right\} \geq \min \{x, v\} \Rightarrow \min \left\{x+y, v+y^{\prime}\right\} \ngtr \min \{x, v\} \text { on } \mathcal{B} \text {. }
$$

Thus, we have established that for games $v, w, w^{\prime} \in \mathcal{G}$ with $v=w-w^{\prime}$ and $x \in F(v)$, $z \in F(w), z^{\prime} \in F\left(w^{\prime}\right)$ with $x=z-z^{\prime}$, the pair $\left(z, z^{\prime}\right)$ is undominated in $\left(w, w^{\prime}\right)$ if, and only

\footnotetext{
${ }^{2}$ These gains are also referred to as 'excesses' in some contributions.
} 
if, (1) holds for all sidepayments $y, y^{\prime}$. This property of $x$ is formalized in the following definition.

Definition 2 For $v \in \mathcal{G}$ and $x \in F(v)$, we call $x$ undominated in $v$ if (1) holds for all sidepayments $y, y^{\prime}$.

Thus, an efficient allocation $x$ in a game $v$ is undominated if it is impossible to redistribute $x$ and at the same time make sidepayments between coalitions in such a way that all coalitions in $\mathcal{B}$ are better off when evaluated in terms of the minimum of their payoffs and their coalitional worths.

\section{Undominated Allocations}

In this section the focus is on undominated allocations in a game. Naturally, the results have direct implications for undominated pairs in an uncertain cooperative game.

\subsection{Characterization of Undominated Allocations}

In order to characterize undominated payoff vectors in a TU game, we begin by stating some preliminary results.

The following lemma is a variation on a result of Derks and Peters (1998) and Zumsteg (1995), and gives an important relation between sidepayments and balanced sets. Its proof is based on Farkas' Lemma.

Lemma 1 Let $\emptyset \neq B \subseteq B^{\prime} \subseteq \mathcal{B}$. Then the following two statements are equivalent.

(a) For every sidepayment $y \in \mathbb{R}^{N}$ with $y(S) \geq 0$ for all $S \in B^{\prime}$ there is an $\hat{S} \in B$ with $y(\hat{S})=0$.

(b) $B^{\prime}$ contains a balanced subset $C$ with $C \cap B \neq \emptyset$.

Proof. Assume that (a) holds and let $y$ and $\hat{S}$ be as in (a). Hence $y \cdot e^{N} \geq 0, y \cdot-e^{N} \geq 0$, $y \cdot e^{S} \geq 0$ for all $S \in B^{\prime}$, and $y \cdot-e^{\hat{S}} \geq 0$. Then Farkas' Lemma implies that $-e^{\hat{S}}$ is a nonnegative weighted sum of the indicator vectors of the coalitions in $B^{\prime}, e^{N}$, and $-e^{N}$. Therefore,

$$
-e^{\hat{S}}=\lambda_{N} e^{N}-\mu_{N} e^{N}+\sum_{T \in B^{\prime}} \lambda_{T} e^{T}
$$


with $\lambda_{N}, \mu_{N}, \lambda_{T}\left(T \in B^{\prime}\right)$ nonnegative. Observe that $\lambda_{N}<\mu_{N}$ and therefore there exist weights $\gamma_{T}\left(T \in B^{\prime}\right)$ with $\gamma_{\hat{S}}>0$ such that

$$
e^{N}=\sum_{T \in B^{\prime}} \gamma_{T} e^{T}
$$

Define $C:=\left\{T \in B^{\prime}: \gamma_{T}>0\right\}$. Then $C$ is balanced and contains $\hat{S}$, hence $C \cap B \neq \emptyset$. This proves (b).

For the converse, let $C$ as in (b) and $y$ a sidepayment with $y(S) \geq 0$ for all $S \in B^{\prime}$. Let $\lambda_{S}>0(S \in C)$ be balancing weights. Then

$$
0=y \cdot e^{N}=y \cdot\left(\sum_{S \in C} \lambda_{S} e^{S}\right)=\sum_{S \in C} \lambda_{S} y(S),
$$

hence $y(S)=0$ for all $S \in C$. This holds in particular for all $S \in C \cap B$.

By taking $B=C$ in (b) of Lemma 1 the following corollary is immediate.

Corollary 1 If $B^{\prime}$ is weakly balanced then for every sidepayment $y$ with $y \geq 0$ on $B^{\prime}$ there is an $S \in B^{\prime}$ with $y(S)=0$.

For an efficient allocation $x$ and a game $v$ we denote by $H(x, v)$ the set of coalitions $S$ that strictly prefer $x$ over their worth in $v$, that is, $x(S)>v(S)$. Further, $E(x, v)$ denotes the set of coalitions, other than $N$, which are indifferent between $x$ and $v$, and $L(x, v)$ denotes the set of coalitions that are worse off with $x$. The sets $H E(x, v)$ and $L E(x, v)$ denote the unions of $H(x, v)$ and $L(x, v)$ with $E(x, v)$, respectively. Note that all these sets are subsets of $\mathcal{B}$, that is, they do not include $N$.

We are now sufficiently equipped to characterize undominated allocations. Let the allocation $x$ in the game $v$ be efficient, that is, $x \in F(v)$. Suppose $x$ is undominated. Then, by (1), for all sidepayments $y$,

$$
\min \{x+y, v\} \geq \min \{x, v\} \Rightarrow \min \{x+y, v\} \ngtr \min \{x, v\} \text { on } \mathcal{B}
$$

and

$$
\min \left\{x, v+y^{\prime}\right\} \geq \min \{x, v\} \Rightarrow \min \left\{x, v+y^{\prime}\right\} \ngtr \min \{x, v\} \text { on } \mathcal{B} \text {. }
$$

The first implication ensures that it is not possible to redistribute the allocation $x$ such that all coalitions in $\mathcal{B}$ are better off in terms of the minimum of their allocation and their worth. The second implication shows that an undominated allocation is robust with respect to a 'sidepayment' perturbation of the game. 
Lemma 2 Let $v \in \mathcal{G}$ and $x \in F(v)$. Then the following statements are equivalent.

(a) $x$ satisfies (2).

(b) Either $H E(x, v) \neq \emptyset$ or $[H E(x, v)=\emptyset$ and $L(x, v)$ is weakly balanced].

Proof. In order to prove the implication $(\mathrm{a}) \Rightarrow(\mathrm{b})$, suppose that $x$ satisfies (2) and $H E(x, v)=\emptyset$. Then for any sidepayment $y$ with $y \geq 0$ on $\mathcal{B},(2)$ implies that $y(S)=0$ for some $S \in \mathcal{B}=L(x, v)$. By Lemma $1, \mathcal{B}$ contains a balanced subset intersecting $L(x, v)$. Since $\mathcal{B}=L(x, v), L(x, v)$ is weakly balanced.

For the converse implication, let $y$ be a sidepayment satisfying the premise in (2). First suppose that $H E(x, v) \neq \emptyset$. Then there is an $S \in \mathcal{B}$ with $x(S) \geq v(S)$. So $\min \{x(S)+y(S), v(S)\} \leq v(S)=\min \{x(S), v(S)\}$, so that (2) holds. Next suppose that $H E(x, v)=\emptyset$ and $L(x, v)=\mathcal{B}$ contains a nonempty balanced collection. Then $\min \{x(S)+y(S), v(S)\} \geq x(S)$ for all $S \in \mathcal{B}$, so by Corollary 1 there is an $S \in \mathcal{B}$ with $y(S)=0$, so that also in this case (2) holds.

The proof of the following lemma is similar and left to the reader.

Lemma 3 Let $v \in \mathcal{G}$ and $x \in F(v)$. Then the following statements are equivalent.

(a) $x$ satisfies (3).

(b) Either $L E(x, v) \neq \emptyset$ or $[L E(x, v)=\emptyset$ and $H(x, v)$ is weakly balanced].

In order to characterize undominated allocations, that is, allocations satisfying (1), we need more than the conjunction of conditions (b) in Lemmas 2 and 3. In particular, we obtain

Theorem 1 An efficient allocation $x$ is undominated in the game $v$ if, and only if, $H E(x, v)$ is weakly balanced or $L E(x, v)$ is weakly balanced.

Proof. Let $x$ be an efficient allocation in the game $v$ satisfying (1). Then $x$ also satisfies (2) and (3), and by Lemmas 2 and 3 the only-if direction is proved if $H E(x, v)$ or $L E(x, v)$ are empty. Suppose therefore that these sets both are nonempty. Also suppose, contrary to what we wish to prove, that neither of them is weakly balanced. Hence by Corollary 1 applied to $L E(x, v)$ there is a sidepayment that is positive on $L E(x, v)$. By multiplying this sidepayment with a sufficiently small positive number we obtain a sidepayment $\hat{y}$ with $\hat{y}(S)>0$ for all $S \in L E(x, v)$ and such that $x(S)+\hat{y}(S)>v(S)$ for all $S \in H(x, v)$. Similarly, we can construct a sidepayment $\tilde{y}$ with $\tilde{y}(S)>0$ for all $S \in H E(x, v)$ and with 
$v(S)+\tilde{y}(S)>x(S)$ for all $S \in L(x, v)$. Thus, on $H E(x, v)$, we have $\min \{x, v\}=v<$ $\min \{x+\hat{y}, v+\tilde{y}\}$, and on $L E(x, v)$, we have $\min \{x, v\}=x<\min \{x+\hat{y}, v+\tilde{y}\}$. This contradicts (1) and completes the proof of the only-if part.

For the if-part, let $y$ and $y^{\prime}$ be sidepayments satisfying the premise of (1), and suppose that $H E(x, v)$ is weakly balanced or $L E(x, v)$ is weakly balanced. If $L E(x, v)=\emptyset$ then $y^{\prime}(S) \geq 0$ for all $S \in \mathcal{B}=H(x, v)$, so weak balancedness of $H(x, v)$ and Corollary 1 imply $y^{\prime}(S)=0$ for some $S \in \mathcal{B}$. Thus, (1) holds. A similar argument holds for the case where $H E(x, v)=\emptyset$. If both $L E(x, v)$ and $H E(x, v)$ are nonempty, then $y \geq 0$ on $L E(x, v)$ and $y^{\prime} \geq 0$ on $H E(x, v)$. Since at least one of these sets is weakly balanced, Corollary 1 implies again (1).

An important consequence of Theorem 1 is the following corollary.

Corollary 2 If $v$ has undominated allocations, then $\mathcal{B}$ is weakly balanced.

It will turn out later that the converse of this corollary also holds; see Theorem 5 in Section 4. Moreover, Corollary 2 implies that for an uncertain cooperative game to have an undominated pair, the set of coalitions $\mathcal{B}$ must be weakly balanced.

\subsection{Stability and Domination}

As is well known since the work of Bondareva (1963) and Shapley (1967), balancedness is closely related to the existence of the core and the anticore of a game. An efficient allocation $x$ is in the [anti]core of the game $v$ if $x(S) \geq[\leq] v(S)$ for all $S \in \mathcal{B}$. A game $v$ is called [anti]balanced if $v(N) \geq[\leq] \sum_{S \in B} \lambda_{S} v(S)$ for every balanced set $B$ with balancing weights $\lambda_{S}, S \in B$. The theorem of Bondareva and Shapley was first formulated for the case of games restricted to a balanced collection $\mathcal{B}$, by Faigle (1989). It states that a game has a nonempty [anti]core if, and only if, it is [anti]balanced. It follows, in particular, that if $\mathcal{B}$ is minimally balanced, then every game $v$ has a nonempty core or a nonempty anticore.

Definition 3 An allocation $x$ in a game $v$ is stable if it is in the core or in the anticore of $v$.

The relation between stability and domination is straightforward.

Theorem 2 Suppose that $\mathcal{B}$ is weakly balanced. Then any stable allocation $x$ in a game $v$ is undominated. 
Proof. If $x$ is stable in $v$, then $\mathcal{B}=H E(x, v)$ or $\mathcal{B}=L E(x, v)$. By weak balancedness of $\mathcal{B}$ and Theorem $1, x$ is undominated.

It easy to construct an example of an undominated allocation in a game $v$ that is not stable, although the game $v$ does have stable allocations. For example, let $N=\{1,2,3\}$, $\mathcal{B}=\{\{1\},\{2\},\{2,3\}\}, v(\{1\})=0, v(\{2\})=2, v(\{2,3\})=0$, and $v(N)=4$. Then $\mathcal{B}$ is weakly balanced and $v$ has a nonempty core. The efficient allocation $(0,1,3)$ is undominated by Theorem 1 but it is not in the core.

\section{Values}

In this section we consider uncertain cooperative games, that is, pairs $\left(w, w^{\prime}\right) \in \mathcal{G} \times \mathcal{G}$, and we investigate the following question. Can we find a value, that is, a mapping $\varphi: \mathcal{G} \rightarrow \mathbb{R}^{N}$ with $\varphi(v) \in F(v)$, such that $\left(\varphi(w), \varphi\left(w^{\prime}\right)\right)$ is an undominated pair for every uncertain cooperative game $\left(w, w^{\prime}\right) \in \mathcal{G} \times \mathcal{G}$ ? A value with this property will be called domination efficient.

\subsection{Minimally Balanced Collections}

We begin by analyzing the case of a minimally balanced collection. It turns out that, in this setting, we can characterize all domination efficient values. Throughout this subsection, $\mathcal{B}$ is a minimally balanced set, say $\mathcal{B}=\left\{S_{1}, \ldots, S_{k}\right\}$, with weight set $\left(\lambda_{S_{j}}\right)_{j=1 \ldots, k}$. That these weights are unique is a well known fact, first proved by Shapley (1967).

Recall from Section 2 that a pair $\left(z, z^{\prime}\right)$ in $F(w) \times F\left(w^{\prime}\right)$ is undominated in $\left(w, w^{\prime}\right)$ if, and only if, $z-z^{\prime}$ is undominated in $w-w^{\prime}$. Since $\mathcal{B}$ is minimally balanced, Theorem 1 implies that either $L\left(z-z^{\prime}, w-w^{\prime}\right)=\emptyset$ or $H\left(z-z^{\prime}, w-w^{\prime}\right)=\emptyset$. In the first case, $z-w \geq z^{\prime}-w^{\prime}$ and in the second case, $z-w \leq z^{\prime}-w^{\prime}$ on $\mathcal{B}$. We have just proved the following lemma.

Lemma 4 Let $\mathcal{B}$ be minimally balanced, and let $\varphi$ be a domination efficient value. Then, for all $w, w^{\prime} \in \mathcal{G}$,

$$
\varphi(w)(S)-w(S) \leq \varphi\left(w^{\prime}\right)(S)-w^{\prime}(S) \text { for all } S \in \mathcal{B}
$$

or

$$
\varphi(w)(S)-w(S) \geq \varphi\left(w^{\prime}\right)(S)-w^{\prime}(S) \text { for all } S \in \mathcal{B}
$$


A set $P \subseteq \mathbb{R}^{k}$ is a monotone path if for every $t \in \mathbb{R}$ there is a unique point $p \in P$ with $\sum_{i=1}^{k} p_{i}=t$ and for all $p, p^{\prime} \in P$ it holds that $p \geq p^{\prime}$ or $p \leq p^{\prime}$.

A monotone $\mathcal{B}$-path is a monotone path $P \subseteq \mathbb{R}^{k}$ satisfying, moreover, that for every $p \in P$ there is an $x \in \mathbb{R}^{N}$ such that, for every $j=1, \ldots, k$, we have $x\left(S_{j}\right)=p_{j} / \lambda_{S_{j}}$. We say that a value $\varphi$ is associated with the monotone $\mathcal{B}$-path $P$ if for every game $v \in \mathcal{G}$ there is a $p \in P$ with $\varphi(v)\left(S_{j}\right)-v\left(S_{j}\right)=p_{j} / \lambda_{S_{j}}$ for every $j=1, \ldots, k$ and $\varphi(v) \in F(v)$. Clearly, since the indicator vectors corresponding to a minimally balanced collection form a linearly independent system, any monotone path $P \subseteq \mathbb{R}^{k}$ is a monotone $\mathcal{B}$-path for a minimally balanced collection of size $k$.

We now obtain

Theorem 3 Let $\mathcal{B}$ be minimally balanced, let $P \in \mathbb{R}^{k}$ be a monotone $\mathcal{B}$-path, and let $\varphi$ be an associated value. Then $\varphi$ is domination efficient.

Proof. Consider two arbitrary games $w, w^{\prime} \in \mathcal{G}$ and define $z=\varphi(w)$ and $z^{\prime}=\varphi\left(w^{\prime}\right)$. It is sufficient to prove that $\left(z, z^{\prime}\right)$ is an undominated pair. Take $x \in F(w)$ and $x^{\prime} \in F\left(w^{\prime}\right)$ and suppose that for every $S \in \mathcal{B}$ we have

$$
\min \left\{x(S)-w(S), x^{\prime}(S)-w^{\prime}(S)\right\} \geq \min \left\{z(S)-w(S), z^{\prime}(S)-w^{\prime}(S)\right\}
$$

It is sufficient to prove that not all these inequalities are strict; in fact, we can demonstrate that all of them are actually equalities. Let $p, p^{\prime} \in P$ correspond to $z, z^{\prime}$ so that $z\left(S_{j}\right)-$ $w\left(S_{j}\right)=p_{j} / \lambda_{S_{j}}$ and $z^{\prime}\left(S_{j}\right)-w^{\prime}\left(S_{j}\right)=p_{j}^{\prime} / \lambda_{S_{j}}$ for every $j=1, \ldots, k$. Without loss of generality, suppose $p^{\prime} \geq p$. Then

$$
\min \left\{x(S)-w(S), x^{\prime}(S)-w^{\prime}(S)\right\} \geq z(S)-w(S)
$$

for all $S \in \mathcal{B}$. This implies $x(S) \geq z(S)$ for all $S \in \mathcal{B}$ and hence

$$
w(N)=x(N)=\sum_{i \in N} \sum_{S \in \mathcal{B}: i \in S} \lambda_{S} x_{i}=\sum_{S \in \mathcal{B}} \lambda_{S} x(S) \geq \sum_{S \in \mathcal{B}} \lambda_{S} z(S)=z(N)=w(N),
$$

hence all inequalities are equalities.

The following converse of Theorem 3 completes the characterization of domination efficient values for the minimally balanced case.

Theorem 4 Let $\mathcal{B}$ be minimally balanced, and let $\varphi$ be a domination efficient value. Then there exists a monotone $\mathcal{B}$-path $P$ to which $\varphi$ is associated. 
Proof. For every $t \in \mathbb{R}$ define the game $v^{t} \in \mathcal{G}$ by $v^{t}(N)=t$ and $v^{t}(S)=0$ for every $S \in \mathcal{B}$, and define the vector $p^{t} \in \mathbb{R}^{k}$ by $p_{j}^{t}=\lambda_{S_{j}} \varphi\left(v^{t}\right)\left(S_{j}\right)$ for every $j=1, \ldots, k$. Then

$$
\sum_{j=1}^{k} p_{j}^{t}=\sum_{j=1}^{k} \lambda_{S_{j}} \varphi\left(v^{t}\right)\left(S_{j}\right)=\varphi\left(v^{t}\right)(N)=t
$$

for every $t \in \mathbb{R}$. Moreover, by Lemma $4, p^{t} \geq p^{s}$ whenever $t \geq s$. Hence $P:=\left\{p^{t}: t \in \mathbb{R}\right\}$ is a monotone $\mathcal{B}$-path.

It suffices to show that $\varphi$ is associated with this path. To this end, let $v$ be an arbitrary game. Lemma 4 implies that for every $t \in \mathbb{R}$ it holds that

$$
\varphi(v)\left(S_{j}\right)-v\left(S_{j}\right) \geq \varphi\left(v^{t}\right)\left(S_{j}\right)-v^{t}\left(S_{j}\right) \text { for all } j=1,2, \ldots, k
$$

or

$$
\varphi(v)\left(S_{j}\right)-v\left(S_{j}\right) \leq \varphi\left(v^{t}\right)\left(S_{j}\right)-v^{t}\left(S_{j}\right) \text { for all } j=1,2, \ldots, k,
$$

that is, that

$$
\varphi(v)\left(S_{j}\right)-v\left(S_{j}\right) \geq p_{j}^{t} / \lambda_{S_{j}} \text { for all } j=1,2, \ldots, k
$$

or

$$
\varphi(v)\left(S_{j}\right)-v\left(S_{j}\right) \leq p_{j}^{t} / \lambda_{S_{j}} \text { for all } j=1,2, \ldots, k .
$$

Because $P$ is a monotone path it follows that $\varphi(v)\left(S_{j}\right)-v\left(S_{j}\right)=p_{j} / \lambda_{S_{j}}$ for some $p \in P$. Moreover, $\varphi(v) \in F(v)$ by definition. Hence, $\varphi$ is associated with $P$.

It should be noted that, if the set of indicator vectors is smaller than $n$, a monotone $\mathcal{B}$-path does not uniquely determine an associated value, since the involved systems of linear equations does not have a unique solution. For instance, let $N=\{1,2,3\}$ and let $\mathcal{B}=\{\{1\},\{2,3\}\}$. Then, in a monotone $\mathcal{B}$-path $P$ with an associated value $\varphi$, only the sum $\varphi_{2}(v)+\varphi_{3}(v)$ is determined through $P$, but not the separate values for players 2 and 3.

\subsection{Existence}

As long as the weakly balanced set $\mathcal{B}$ determines a linearly independent set of characteristic vectors, monotone $\mathcal{B}$-paths with associated values can be constructed as in the preceding subsection. These associated values are again domination efficient. For example, suppose $N=\{1, \ldots, 4\}$ and let

$$
\mathcal{B}=\{\{1,2\},\{3,4\},\{1,3\},\{2,4\}\}
$$


This set is not minimally balanced, but the construction used in the proof of Theorem 4 is still possible. It is not clear what a complete characterization of all domination efficient values would look like without the minimal-balancedness assumption. We can, however, completely settle the existence question regarding domination efficient values. Observe that it is easy to construct undominated allocations from a domination efficient value. Hence, from the remark following Corollary 2, we already know that $\mathcal{B}$ has to be weakly balanced in order to ensure that a domination efficient value exists. The following theorem demonstrates that weak balancedness is also sufficient for existence. Additionally, this implies the existence of undominated allocations in certain games.

Theorem 5 If $\mathcal{B}$ is weakly balanced, there exists a domination efficient value.

Proof. Because $\mathcal{B}$ is weakly balanced, there exists a nonempty minimally balanced subcollection $\mathcal{C} \subseteq \mathcal{B}$. Define a monotone $\mathcal{C}$-path as in the previous subsection and let $\varphi$ be a value associated with this path. This implies that we have

$$
\varphi(v)(S)-v(S) \geq \varphi(w)(S)-w(S) \text { for all } S \in \mathcal{C}
$$

or

$$
\varphi(v)(S)-v(S) \leq \varphi(w)(S)-w(S) \text { for all } S \in \mathcal{C} .
$$

This implies that, for all $v, w \in \mathcal{G}$, there do not exist $x \in F(v)$ and $y \in F(w)$ such that

$$
\min \{x(S)-v(S), y(S)-w(S)\}>\min \{\varphi(v)(S)-v(S), \varphi(w)(S)-w(S)\}
$$

for all $S \in \mathcal{C}$. Because $\mathcal{C} \subseteq \mathcal{B}$, this immediately implies that there do not exist $x \in F(v)$ and $y \in F(w)$ such that $(4)$ holds for all $S \in \mathcal{B}$. Therefore, $\varphi$ is domination efficient.

\subsection{More Than Two States}

As mentioned in Section 2, the definition of a domination efficient value can be modified by employing an alternative notion of dominance that is not restricted to two states. Specifically, we could require the following.

Definition 4 For $m \geq 2, w^{1}, \ldots, w^{m} \in \mathcal{G}$, and $q^{j}, z^{j} \in F\left(w^{j}\right)(j=1, \ldots, m)$, we say that the $m$-tuple $\left(q^{1}, \ldots, q^{m}\right)$ generalized dominates the $m$-tuple $\left(z^{1}, \ldots, z^{m}\right)$ if for every $S \in \mathcal{B}$ we have

$$
\min \left\{q^{j}(S)-w^{j}(S): j=1, \ldots, m\right\}>\min \left\{z^{j}(S)-w^{j}(S): j=1, \ldots, m\right\} .
$$

If there is no $m$-tuple $\left(q^{1}, \ldots, q^{m}\right)$ generalized dominating $\left(z^{1}, \ldots, z^{m}\right)$ then the latter $m$-tuple is called generalized undominated in $\left(w^{1}, \ldots, w^{m}\right)$. 
Accordingly, a generalized domination efficient value can be defined. Clearly, a generalized domination efficient value is a domination efficient value. Furthermore, because the values of Theorems 3 and 5 satisfy this stronger notion of domination efficiency, all results of this section can be generalized by allowing for an arbitrary number of possible states. Therefore, we obtain the following corollaries to Theorems 3 to 5 .

Corollary 3 If $\mathcal{B}$ is weakly balanced, there exists a generalized domination efficient value.

Corollary 4 Let $\mathcal{B}$ be minimally balanced, and let $\varphi$ be a generalized domination efficient value. Then there exists a monotone $\mathcal{B}$-path $P$ to which $\varphi$ is associated.

Corollary 5 Let $\mathcal{B}$ be minimally balanced, let $P \in \mathbb{R}^{k}$ be a monotone $\mathcal{B}$-path, and let $\varphi$ be an associated value. Then $\varphi$ is generalized domination efficient.

\section{Concluding Remarks}

This paper provides an analysis of domination efficiency defined for TU games. A related question is what happens if our notion of domination is modified by requiring weak inequalities with at least one strict inequality instead of the strict inequalities in Definition 1. Many of the results in this paper can be modified quite easily to apply to this alternative framework of strongly undominated allocations and strong domination efficiency as well. In particular, an analogue of Theorem 1 can be established, and an equivalence rather than an implication regarding stable allocations and strongly undominated allocations can be derived. Furthermore, the characterization of values associated with monotone $\mathcal{B}$-paths remains to be valid in the case of minimally balanced collections. The most important difference is that the existence issue is an open problem. Balancedness of $\mathcal{B}$ is necessary for the existence of strongly domination efficient values but it is not sufficient. This alternative model and its properties is an issue to be explored in future work. 


\section{References}

O.N. Bondareva (1963), Some applications of linear programming methods to the theory of cooperative games, Problemy Kibernetiki 10, 119-139.

W. Bossert, E. Nosal and V. Sadanand (1996), Bargaining under uncertainty and the monotone path solutions, Games and Economic Behavior 14, 173-189.

W. Bossert and H. Peters (2001a), Minimax regret and efficient bargaining under uncertainty, Games and Economic Behavior 34, 1-10.

W. Bossert and H. Peters (2001b), Efficient solutions to bargaining problems with uncertain disagreement points, Social Choice and Welfare, forthcoming.

J. Derks and H. Peters (1998), Ordering, excess functions, and the nucleolus, Mathematical Social Sciences 36, 175-182.

U. Faigle (1989), Cores of games with restricted cooperation, Zeitschrift für Operations Research 33, 405-422.

L.S. Shapley (1967), On balanced sets and cores, Naval Research Logistics Quarterly 14, 453-460.

S.M. Zumsteg (1995), Non-cooperative aspects of cooperative game theory and related computational problems, PhD Thesis, Zürich. 